\title{
MILP for selecting portfolio of $R \& D$ projects in public organizations with partial and full resource allocation policies
}

\author{
Nancy Maribel Arratia Martínez ${ }^{1}$ Fernando López Irarragorri $^{1}$ \\ ${ }^{1}$ Autonomous University of Nuevo León, San Nicolás de los Garza, México. \\ nancyamtz@gmail.com, flopez65@gmail.com
}

\begin{abstract}
In general, the problem of $R \& D$ project portfolio selection (RDPPS) is to choose a set of project proposals that optimize certain impact measures designated by the decision maker.

In this paper we present a MILP model, that incorporates the most relevant aspects of the problem found in the literature, that supports both full (all or nothing) and partial (a certain amount between a minimum and a maximum value) resource allocation policies to projects. In most of the reviewed papers about RDPPS full allocation resources policies are implemented, a few implement partiall allocation policies, but most of them presents very simple models.
\end{abstract}

Keywords: Project portfolio selection, R\&D projects, mixed integer linear programming.

\section{Introduction}

The problem of $R \& D$ project portfolio selection (RDPPS) is to choose a set of project proposals that optimize certain impact measures designated by the decision maker. The proposals are characterized by one or more social objectives and geographic regions within a certain area of influence [6]. A project can be broken down into tasks to which objectives, goals and resources requirements are linked [10].

A classification of R\&D Project Portfolio Selection Problem given in Bard et al. [1] distinguishes two classes: static and dynamic. The static class is referred to those situations in which all funded project proposals begins and finish at a certain common date, while the dynamic class is referred to a portfolio selection process where projects proposals can be inserted or withdrawn at any moment in time. In this work the static class of R\&D Project portfolio selection is addressed.

We propose a Multiobjective Mixed Integer Lineal Mathematical Model that allows the representation of R\&D projects by its tasks, allocation of resources to tasks, and interdependencies among tasks and projects. Our model, supports both partial (assigning any amount between a minimum and a maximum quantity) and full allocation policies (assigning all or nothing of a requested quantity) of resources to tasks. Most relevant papers dealing with RDPPS implement a full allocation resource policy and employs eiher Data Envelopment Analisys (most dominant method), Heuristic or Metha-heuristic methods, or Analitical methods (very few) for selecting the portfolio. Full allocation policies imply the representation of projects by binary variables in any of those methods mentioned above. And henceforth impact meassures for the portfolio are represented as sum of products of impact coefficients (cost, resources assigned, evaluation, etc.) and a linear function of the binary variable which represent the proyect. When resources are in nature monolitic (team, a machine, an installation, etc.) full allocation policies works well because there is not other decision possible that allocate or not, but when resources can be tear down (like money, material constructions, gas, etc.) then we will show in this paper that it best to implement partial allocation policies than full policies.

This paper is organized as follows, first we present relevant aspects of the RDPPS problem and implications of using full or partial allocation resource policies are discused (section 2). Then we make a brief mention of state of art of RDPPS (section 3), thereafter the mathematical model is presented (section 4). Also some experimental results are presented (section 5) and finally conclusions and future work are discussed (section 6).

\section{Problem description}

The static RDPPS problem, as other Project portfolio problems do, have some relevant characteristics that must be represented in any model [5]:

1. Risk in achieving portfolio impacts.

2. Balancing portfolio policies.

3. Interdependencies among task or projects

4. Break down projects into tasks.

5. Resource allocation policies.

6. Projects and task scheduling.

(1) to (3) are typically addressed in most papers related to RDPPS. (4) and (5) are less likely addressed and (6) is mainly treated as a standalone problem. 
In Litvinchev et al. [10] can be found a discussion about (4). In this paper we do not address (6), and our main concern is (5).

In was follows we will discuss the impact of resource allocation policies in RDPPS.

\subsection{Partial and full resources allocation to tasks or projects.}

Most of the work presented in the area of selection of project portfolios dealing with discrete resource allocation, by this we referring to those resources on which they choose to be supported or not supported (the project or tasks). However, there are situations where resources are continuous in nature. That is, the decision of allocation not only incorporates the actions of support or not support, but rather allows partial allocation consistent with allocate an amount of resources within a minimum and a maximum value.

In the proposed mathematical model, to represent partial allocation, we defined continuous variables representing the amount of resources selected to support each project tasks. Each variable is bounded by a minimum and a maximum amount of resources needed to carry out each task. So that the sum of the resources allocated to each of the tasks in a project form the amount of resources allocated to the project, that amount is also bounded by minimum and maximum amount of resources needed to carry out the project.

\section{Some background}

Portfolio Management was first applied in capital budgeting, asset investment, in the 50's and has evolved through the 70's to become a useful planning tool.

Then, between the 80's and 90's the use of portfolio management is extended for the selection of new products and the allocation of resources in research and development (Dickinson et al. [4]). Such is the case of Ghasemzadeh, F., \& Archer, N. P. [8], they integrate a methodology for selecting portfolios by taking advantage of the most useful features of some existing methods and implemented it in a prototype (PASS: Project Analysis and Selection System) used as a tool to support decision making. The proposal consists of five processes: prescreening, individual project analysis, selection, optimal portfolio selection and adjustment. The methodology have taken into account the uncertainty and risk, but assumed that the parameters could be approximated accurately.

Also, Huchzermeier, A., \& Loch, C. H. [9] present a dynamic programming model of real options where the risk is treated by five types of operational uncertainties (price or sales forecast, cost, time, performance, market requirements). And then Santiago, L. P., \& Bifano, T. G.
[13] show an application to the model, in the development of a new high-tech product.

A more general approach was constructed by Stummer, C. and Heidenberger, K. [14]: reducing the number of projects, obtaining the solution space of efficient portfolios by integer linear programming model and the decision maker is guided by an interactive system for securing preferences render correctly. The main limitation is undoubtedly the amount of thirty projects that can be handled in the second and third phases.

Carazo, A. F., Gómez, T., Molina, J., Hernández-Díaz, A. G., Guerrero, F. M., \& Caballero, R. [2] developed a multiobjective mixed integer non-linear programming model for project portfolio selection and scheduling problem tailored metaheuristic for solving instances with about hundred of projects. The model also allowed for incorporating interdependencies among projects. The weakness of their approach lies in the "all or nothing" allocation policy of resources, and in the fact that the model wasn't lineal.

After an extensive literature review about R\&D project portfolio selection we realize that there are only a few published works dealing with very large scale problems. Among these Litvinchev, I. S., López, F., Alvarez, A., \& Fernández, E. [11] developed a bi-objective mixed integer linear programming model, in which they implemented partial allocation of resources. They solve instances up to 25,000 projects in a few seconds. But the main limitations of this work were the assumption of independencies among projects.

In conclusion, early evidence shows that there is a wide variety of methodologies developed to address the problem of portfolio selection, each with specific techniques for the treatment of the most important characteristics of the project portfolio selection problem, but with respect to the number of projects that are managed in the experimental part, there are few studies that successfully solved instances with more than 100 projects and very few implemented flexible policies for allocating resources.

\section{Proposed mathematical model}

To state the model mathematically, we define first the following notation.

\subsection{Sets and parameters}

$J$ : Projects competing for financial support, $j=1,2,3, \ldots,|J|$.

$K$ : Areas of research and development, its indices $k=1,2,3, \ldots,|K|$.

$J_{k}$ : Projects belonging to area $k$.

$I$ : Tasks $i=1,2,3, \ldots|I|$.

$C$ : Synergies, $S=\{1,2,3, \ldots|S|\}$.

$C^{s}$ : Set of elements (pairs) of synergy $\mathrm{s}$,

$C^{s}=\left\{\left(j_{1}, i_{1}\right),\left(j_{2}, i_{2}\right), \ldots,\left(j_{\left|C^{s}\right|}, i_{\left|C^{s}\right|}\right)\right\}$. 
$B$ : Set of benefit type synergies, $B \subset C$.

$L$ : Synergies of resource consumption reduction, $L \subset C$.

$H$ : Synergies of enlargement of resource consumption, $H \subset C$.

$\tau$ :Technical synergies, $T \subset \tau$ is defined as a set of indices synergies which in turn belong to a technical synergy, $T=\left\{s_{1}, s_{2}, \ldots, s_{|T|}\right\}$.

$\eta^{s}$ :Synergetic effect of resource consumption reduction by s.

$\lambda^{s}$ : Synergetic effect of enlargement of resource consumption by $s$.

$v^{s}$ : Synergetic effect of benefit by s.

$w_{j}$ : The social impact of the project $\mathrm{j}$.

$\rho_{j i}$ : Relative importance of the task $\mathrm{i}$ of the project $\mathrm{j}$.

$P_{G}:$ Available budget.

$R_{j i}^{-}, R_{j i}^{+}$: Minimum and maximum amount to support the task j,i.

$P_{k}^{-}, P_{k}^{+}$:Minimum and maximum amount of resources to the area $\mathrm{k}$.

$M_{j}^{-}, M_{j}^{+}$:Minimum and maximum amount to support the project j.

$m^{s+}, m^{s-}$ :Minimum and maximum number of tasks to enable synergy s.

$E_{T}^{-}, E_{T}^{+}$:Minimum and maximum number of synergies to enable technical synergy $\mathrm{T}$.

\subsection{Variables}

Use $x_{j i}$ Amount of resource assigned to task $\mathrm{i}$ of project j.

$y_{j}=\left\{\begin{array}{l}1, \text { the project } \mathrm{j} \text { is enough supported. } \\ 0, \text { d.o.m. }\end{array}\right.$

$z_{j i}=\left\{\begin{array}{l}1, \text { the task } \mathrm{j}, \mathrm{i} \text { is enough supported. } \\ 0, \text { d.o.m. }\end{array}\right.$

$\sigma_{1}^{s}=\left\{\begin{array}{l}1, \text { synergistic set has cardinality } \geq m^{s-} \\ 0, \text { d.o.m. }\end{array}\right.$

$\sigma_{2}^{s}=\left\{\begin{array}{l}1, \text { synergistic set has cardinality } \leq m^{s+} \\ 0, \text { d.o.m. }\end{array}\right.$

$\sigma^{s}=\left\{\begin{array}{l}1, \text { synergy s is activated, } \sigma^{s}=\sigma_{1}^{s}+\sigma_{2}^{s}-1 \\ 0, \text { d.o.m. }\end{array}\right.$

\subsection{Objectives}

Two objectives are considered: (1) represent the impact of the portfolio in a similar way as proposed in Litvinchev et al.[11], (2) represent the total amount of supported projects, it is also typical to use a threshold.

$$
\begin{gathered}
\sum_{j \in J} w_{j}\left(\sum_{i \in I}\left(a_{j i} z_{j i}+b_{j} x_{j i}\right) \cdot \rho_{j i}\right)+\sum_{s \in B} v^{s} \sigma^{s} \\
\sum_{j \in J} y_{j}
\end{gathered}
$$

We choose to consider the amount of funded projects as an objective because when we have two portfolios with equally high quality and no difference in resources, it is usually desirable to choose the one with more projects.

The parameters $a_{j i}$ and $b_{j i}$ are defined in the same way as in Litvinchev et al. (2010).

$$
a_{j i}=\alpha_{j i}-\frac{R_{j i}^{-}\left(1-\alpha_{j i}\right)}{R_{j i}^{+}-R_{j i}^{-}}, \quad b_{j i}=\frac{\left(1-\alpha_{j i}\right)}{R_{j i}^{+}-R_{j i}^{-}}
$$

If $R_{j i}^{-}$and $R_{j i}^{+}$are very closer then the objective (1) have to be modified, the expression $\left(a_{j i} z_{j i}+b_{j} x_{j i}\right)$ should change to $\left(x_{j i} \cdot \frac{1}{R_{j i}^{+}}\right)$.

\subsection{Constraints}

The constraints of the model are:

$$
\begin{aligned}
& \sum_{j \in J} \sum_{i \in I} x_{j i} \leq P_{G} \\
& P_{k}^{-} \leq \sum_{j \in J_{k}} \sum_{i \in I} x_{j i} \leq P_{k}^{+}, \quad k \in K \\
& M_{j}^{-} y_{j} \leq \sum_{i \in I} x_{j i} \leq M_{j}^{+} y_{j}, \quad j \in J \\
& R_{j i}^{-} z_{j i} \leq x_{j i} \leq R_{j i}^{+} z_{j i}, \quad\left\{\begin{array}{l}
i \in I, j \in J \\
(j, i) \notin L \cup H
\end{array}\right. \\
& y_{j} \leq \sum_{i \in I} z_{j i}, \quad j \in J \\
& \sum_{i \in I} z_{j i} \leq|I| y_{j}, \quad j \in J \\
& \sum_{(j, i) \in C^{s}} z_{j i}-m^{s-}+1 \leq\left|C^{s}\right| \sigma_{1}^{s}, s \in C \\
& \left|C^{s}\right| \sigma_{1}^{s} \leq \sum_{(j, i) \in C^{s}} z_{j i}-m^{s-}+\left|C^{s}\right|, s \in C \\
& m^{s+}-\sum_{(j, i) \in C^{s}} z_{j i}+1 \leq\left|C^{s}\right| \sigma_{2}^{s}, s \in C \\
& \left|C^{s}\right| \sigma_{2}^{s} \leq m^{s+}-\sum_{(j, i) \in C^{s}} z_{j i}+\left|C^{s}\right|, s \in C \\
& \sigma^{s}=\sigma_{1}^{s}+\sigma_{2}^{s}-1, \quad s \in C \\
& \sum_{(j, i) \in C^{s}} x_{j i} \leq \sum_{(j, i) \in C^{s}} R_{j i}^{+} z_{j i}-\eta^{s} \sigma^{s}, s \in L
\end{aligned}
$$




$$
\begin{aligned}
& \sum_{(j, i) \in C^{s}} x_{j i} \geq \sum_{(j, i) \in C^{s}} R_{j i}^{-} z_{j i}+\lambda^{s} \sigma^{s}, s \in H \\
& \sum_{(j, i) \in C^{s}} x_{j i} \geq \sum_{(j, i) \in C^{s}} R_{j i}^{-} z_{j i}-\eta^{s} \sigma^{s}, s \in L \\
& \sum_{(j, i) \in C^{s}} x_{j i} \leq \sum_{(j, i) \in C^{s}} R_{j i}^{+} z_{j i}+\lambda^{s} \sigma^{s}, s \in H \\
& E_{T}^{-} \leq \sum_{s \in T} \sigma^{s} \leq E_{T}^{+}, \quad T \in \tau \\
& x_{j i} \geq 0, y_{j}, z_{j i}, \sigma^{s}, \sigma_{1}^{s}, \sigma_{2}^{s} \in\{0,1\}
\end{aligned}
$$

Constraints (4-7) are the typical budged constraints, defined at portfolio, area, project and task levels respectively. Constraints (8-9) relate tasks to projects. All these constraints are defined in Litvinchev et al. [10].

Constrains (10-14) represent the activation of synergies. With the particularity that synergies are represented at the tasks level, this offer much more flexibility in defining synergies than represent these at a project level. Here a synergy is activated when a subset of task in $C^{s}$ are funded with cardinality less than a number $m^{s+}$ and greater than a $m^{s-}$ see also A. F. Carazo et al. [2,3].

If it is $m^{s+}$ and $m^{s-}$ equal, it is said that the set that produces the synergistic effect has strictly a cardinality $m^{s}$, where $m^{s}=m^{s-}=m^{s+}$. In (10-13) $\sigma_{1}^{s}$ and $\sigma_{2}^{s}$ indicate that you meet the lower and upper limits respectively. We define $\sigma^{s}$ in terms of the previous two to verify that the synergy $s$ is activated. $\sigma_{1}^{s}$ and $\sigma_{2}^{s}$ can not be zero simultaneously, since that would imply that the sum $\sum_{(j, i) \in C^{s}} z_{j i}$ is less than $m^{s-}$ and over $m^{s+}$ and this is impossible because $m^{s-} \leq m^{s+}$. Moreover, our formulation for the restriction (19) eliminates a major weakness of the model A. F. Carazo et al. [2,3], the non-linearity. Constraints (15-18) represent resources synergies.

Technical synergies are defined to keep control over the synergies of tasks that could be activated. They work limiting the number of active synergies from a group of them. We call $T$ the set containing the indices of synergies to be restricted, i.e., $T=\left\{s_{1}, s_{2}, s_{3}, \ldots, s_{|T|}\right\}$, so that only a number less than or equal to $E_{T}^{+}$and / or greater than or equal to $E_{T}^{-}$of them can be active. The set of all $T$ is $\tau$. In our model technical constraints are represented by constraint (19). This representation allows incorporating more than two groups of mutually exclusive groups of tasks.

\section{Others constraints.}

In some cases, it is desirable to balance the portfolio for the number of projects supported by area. That is,

$$
d_{j}^{-} \leq \sum_{j \in J_{k}} y_{j} \leq d_{j}^{+}
$$

where $d_{j}^{-} \quad \mathrm{y} \quad d_{j}^{+}$are respectively lower and upper bounds.

\section{Experimentation}

In [5], we showed the efficiency of the proposed model by conducting an experiment on instances taken from a previously published paper and randomly generated very large scale instances, with computing times of the worst cases less than 30 minutes. Being one of a very few papers that employ instances with 100 o more projects in numerical experiments, see F. López and N.M. Arratia [5].

In this paper, we present the results obtained by studying the effect produced in the performance of the model and in obtaining solutions to solve randomly generated instances. For each instance we reduce the range associated with minimum and maximum amounts of resources to be allocated for each task and project (by shifting the minimum toward the maximum value). For that we employ different levels of reduction: $10 \%, 30 \%$, $50 \%, \quad 90 \%, \quad 95 \%, \quad 99 \%$ and $100 \%$. The case of $100 \%$ reduction is equivalent to consider equal minimum and maximum values of the range by activity and project.

The reductions were carried out considering different percentages of the number of proposed projects in each instance: $25 \%, 50 \%, 75 \%$ and $100 \%$ of projects. In the case of $100 \%$ reduction, only considered the case of $100 \%$ reduction projects.

The group of instances (15 instances) that we employ has the follow characteristics: 100 projects, 5 tasks, 2 areas, 0 synergies.

Tables 1 and 2, shows the mean percentage of reduction in projects and impact in the obtained solution when compared to the original problem without any reduction.

\begin{tabular}{|c|rrrrrr|}
\hline \multicolumn{7}{|c|}{ Percent of reduction in the number of projects } \\
\hline $\begin{array}{c}\text { Percent of } \\
\text { projects } \\
\text { with reduc- } \\
\text { tion }\end{array}$ & $10 \%$ & $30 \%$ & $50 \%$ & $90 \%$ & $95 \%$ & $99 \%$ \\
\hline $25 \%$ & 0.9 & 2.55 & 4.97 & 8.01 & 8.01 & 8.18 \\
$50 \%$ & 1.83 & 5.65 & 9.61 & 16.2 & 16.2 & 15.9 \\
$75 \%$ & 2.65 & 8.6 & 15 & 24.3 & 24.7 & 24.7 \\
$100 \%$ & 4.46 & 11.6 & 20 & 31.9 & 32.3 & 32.7 \\
\hline
\end{tabular}

Table 1. Results obtained by reducing the range that bounds the amount of resources that are allocated to tasks.

With $100 \%$ reduction in the range associated with the allocation of resources to tasks, we obtained an average reduction in the number of projects of $5.32 \%$ and $2.61 \%$ 
in the impact measure compared to the solutions obtained with the original instances without reduction.

Also original instances were solved considering only total allocation, and as expected the solution match with that obtained in the case of $100 \%$ reduction, that with respect to the number of projects and the measure of impact.

In general, when reviewing the results, it appears that increasing the percentage reduction and the number of projects that are reduced the lesser projects can be funded, and the lesser is the impact of portfolio. Also the computing time increases (see Table 3). This presumption was proved statistically.

\begin{tabular}{|r|rrrrrr|}
\hline \multicolumn{1}{|c|}{ Percent of reduction in the impact measure } \\
\hline $\begin{array}{c}\text { Percent of } \\
\text { projects with } \\
\text { reduction }\end{array}$ & $10 \%$ & $30 \%$ & $5 \%$ & $90 \%$ & $95 \%$ & $99 \%$ \\
\hline $25 \%$ & 0.41 & 1.05 & 1.73 & 3.81 & 3.83 & 3.86 \\
$50 \%$ & 0.82 & 2.21 & 3.69 & 7.57 & 7.65 & 7.51 \\
$75 \%$ & 1.23 & 3.3 & 5.87 & 10.8 & 11 & 11 \\
$100 \%$ & 2.7 & 4.38 & 7.54 & 13.5 & 13.7 & 13.8 \\
\hline
\end{tabular}

Table 2. Percent reductions in the impact measure of solutions with reduction in the range that bounded the amount of resources that are allocated to tasks.

\begin{tabular}{|r|rrrrrr|}
\hline \multicolumn{7}{|c|}{ Average time of solution } \\
\hline $\begin{array}{c}\text { Percent of } \\
\text { projects with } \\
\text { reduction }\end{array}$ & $10 \%$ & $30 \%$ & $50 \%$ & $90 \%$ & $95 \%$ & $99 \%$ \\
\hline $25 \%$ & 1.15 & 1.1 & 1.22 & 1.07 & 1.03 & 1.01 \\
$50 \%$ & 1.19 & 1.33 & 1.33 & 1.11 & 1.05 & 1.04 \\
$75 \%$ & 1.41 & 1.5 & 2.12 & 1.32 & 1.58 & 1.41 \\
$100 \%$ & 1.36 & 1.78 & 2.52 & 2.62 & 4.38 & 7.88 \\
\hline
\end{tabular}

Table 3. Results of average time.

\section{Conclusions}

In this paper, is proposed a Multiobjective Mixed Integer Lineal Mathematical Model that allow the representation of R\&D projects by its tasks, the definition of interdependencies among tasks or projects, and allows partial and full allocation of resources to tasks. Characteristics of the RDPPS than can not be found together on previous works.

The paper presented some of the results of experiments conducted to observe the effects on the solutions obtained and the performance of the model using partial allocation of resources and studying its transformation to the case of full allocation.

Those conduce us to the conclusion that partial allocation should be employed whenever is possible, yielding more funded projects and best impact of the portfolio.

As future work we will executing more large experiments, and will develop a full resource policy only model to compare against the one being presented in this paper.

\section{References}

[1] Bard, J. F., Balachandra, R., \& Kaufmann, P. E. , “An interactive approach to $\mathrm{R} \& \mathrm{D}$ project selection and termination", Engineering Management, IEEE Transactions on, 35(3), 139-146, 1988.

[2] Carazo, A. F., Gómez, T., Molina, J., HernándezDíaz, A. G., Guerrero, F. M., \& Caballero, R., "Solving a comprehensive model for multiobjective project portfolio selection", Computers \& operations research, 37(4), 630-639, 2010.

[3] Carazo, A. F., Gómez, T., \& Pérez, F., “Análisis de los principales aspectos que afectan la decisión de selección y planificación de carteras e proyectos", Rect@, 2012.

[4] Dickinson, M. W., Thornton, A. C., \& Graves, S., "Technology portfolio management: optimizing interdependent projects over multiple time periods", Engineering Management, IEEE Transactions on, 48(4), 518-527, 2001.

[5] E. Fernández, F. Lopez, and J. Navarro, "Decision support tools for R\&D project selection in public organizations," in Proc. IAMOT, Washington, DC, 2004.

[6] E. Fernández, Cervantes, E. L., Castillo, J. N., \& López, I. V., “Aplicación de metaheurísticas multiobjetivo a la solución de problemas de cartera de proyectos públicos con una valoración multidimensional de su impacto", Gestión y Política Pública, 20(2), 381-432, 2011.

[7] F. López Irarragori and N. M. Arriata Martínez, "R\&D project portfolio selection in public organizations", Technical Report PISIS-2013-01, Graduate Program in Systems Engineering, UANL, San Nicolás de los Garza, México, March 1st, 2013.

[8] Ghasemzadeh, F., \& Archer, N. P., "Project portfolio selection through decision support.", Decision Support Systems, 29(1), 73-88, 2000.

[9] Huchzermeier, A., \& Loch, C. H., "Project Management Under Risk: Using the Real Options Approach to Evaluate Flexibility in R... D", Management Science, 47(1), 85-101, 2001.

[10] Litvinchev, I., López, F., Escalante, H. J., \& Mata, M. , "A milp bi-objective model for static portfolio selection of R\&D projects with synergies", Journal of Computer and Systems Sciences International, 50(6), 942-952, 2011.

[11] Litvinchev, I. S., López, F., Alvarez, A., \& Fernández, E. , “ Large-scale public R\&D portfolio selection by maximizing a biobjective impact measure", Systems, Man and Cybernetics, Part A: Systems and Humans, IEEE Transactions on, 40(3), 572-582, 2010.

[12] Mait Rungi, " Visual representation of interdependencies between projects", Computers and Industrial 
Engineering, 1061-1072, Alexandria, Egipt, October 2007.

[13] Santiago, L. P., \& Bifano, T. G., “ Management of R\&D projects under uncertainty: A multidimensional approach to managerial flexibility", Engineering Management, IEEE Transactions on, 52(2), 269-280, 2005.
[14] Stummer, C., \& Heidenberger, K., "Interactive R\&D portfolio analysis with project interdependencies and time profiles of multiple objectives", Engineering Management, IEEE Transactions on, 50(2), 175-183, 2003. 